\title{
Pilotprojekt für die Behandlung arabischsprachiger Flüchtlinge
}

Der Freie Verband Deutscher Zahnärzte (FVDZ) startet ein Pilotprojekt zur Flüchtlingshilfe: Zur Unterstützung der zahnärztlichen Kollegen richtet der FVDZ eine Dolmetscher-Hotline für Flüchtlinge ein. Dies hat der Bundesvorstand im Vorfeld der diesjährigen Hauptversammlung in Bonn beschlossen. „Wir erleben täglich in unseren Praxen, wie schwer es ist, Flüchtlinge und Asylbewerber fachgerecht zu behandeln. Die Sprachbarriere stellt dabei für uns das größte Problem dar“, erklärte der stellvertretende Bundesvorsitzende Joachim Hoffmann den Hintergrund des Projekts.Nur wenige Flüchtlinge aus dem arabischen Raum sprechen Englisch oder Deutsch. Eine Verständigung ist daher für die Zahnärzte in vielen Fällen unmöglich. Während es in den Großstädten wie Berlin, Hamburg, Köln, Frankfurt oder München arabisch sprechende Dolmetscher gibt, ist es im ländlichen Bereich sehr schwierig,jemanden mit diesen Übersetzungsfähigkeiten zu finden. Für den FVDZ wird nun zunächst für 2 Monate eine syrische Zahnärztin aus Niedersachsen, die mehrere arabische Dialekte beherrscht, „Erste Hilfe“ bei Verständigungsschwierigkeiten leisten. Um rechtliche Hürden zu minimieren, hat der FVDZ einen Leitfaden zur Behandlung von Flüchtlingen entwickelt. „Mit dem Pilotprojekt wagen wir uns auf unbekanntes Terrain, aber wir haben erkannt, dass wir handeln müssen“, sagte die FVDZ-Bundesvorsitzende Kerstin Blaschke. Der stellvertretende FVDZ-Bundesvorsitzende Dr. Michael Betz betonte: „Mit dieser Unterstützung der Kollegen können wir unsere soziale Verantwortung nach außen tragen und eine pragmatische Lösung anbieten.“

Sobald die Hotline freigeschaltet ist, wird dies auf der Verbandshomepage unter www.fvdz.de veröffentlicht. Nach einer 2-monatigen Erprobung soll der Dolmetscherdienst evaluiert und bei positiver Resonanz verlängert werden. 